Como citar este artigo: Morais AHA. Proteinas bioativas das sementes de tamarindo reduzem leptina plasmática independente da perda de peso em ratos com obesidade. Revista Saúde (Sta. Maria). 2018; 44(2).

Autor correspondente: Ana Heloneida de Araújo Morais Graduação em Nutrição (2000), mestrado em Bioquímica (química e função de proteínas) pela Universidade Federal do Rio Grande do Norte (2002) e doutorado em Biologia molecular (nutrigenômica) pela Universidade de Brasilia (2007) Email para contato:

aharaujomorais@gmail.com

Data de Submissão: 14/06/18

Data de aceite: 10/07/2018

Conflito de Interesse: Não há conflito de interesse

(cc) $\mathrm{BY}-\mathrm{NC}-\mathrm{ND}$

\section{Proteínas bioativas das sementes de tamarindo reduzem leptina plasmática independente da perda de peso em ratos com obesidade}

\author{
Izael de Sousa Costa; Fabiana Maria Coimbra de Carvalho; Vanessa Cristi- \\ na Oliveira Lima; Amanda Fernandes de Medeiros; Alexandre Coelho Serquiz; \\ Luiza Gabriella Soares Dantas Pinheiro; Bruna Leal Lima Maciel; Elizeu Antu- \\ nes dos Santos; Ana Heloneida de Araújo Morais.
}

\section{RESUMO}

Introdução: A obesidade caracteriza-se pelo acúmulo excessivo de gordura corporal, sendo essa responsável por elevadas concentrações de leptina. Pesquisas têm procurado adjuvantes no seu tratamento e as proteínas isoladas das sementes de tamarindo tem se destacado por possuírem atividades anti-obesidade e anti-inflamatória. Objetivos: Avaliar o efeito das proteínas de tamarindo sobre o peso, o índice de Lee e a concentração plasmática de leptina em ratos Wistar com obesidade. Metodologia: Utilizaram-se três grupos $(n=5)$ de ratos Wistar, adultos, machos e com obesidade (Índice de Lee $\geq 300$ ). As proteínas isoladas foram oferecidas durante 10 dias, por gavagem, aos animais do grupo teste. Ao grupo com tratamento convencional foi ofertada uma dieta padrão. Os animais do terceiro grupo receberam dieta de alto índice glicêmico e carga glicêmica, a mesma utilizada para indução da obesidade. No primeiro e no décimo dia de experimento, aferiu-se o peso e foi medido o comprimento caudal dos animais para avaliação do índice de Lee. Após a eutanásia, coletou-se sangue para dosagem de leptina. Resultados: $O$ índice de Lee e o peso não sofreram influência em ambos os tratamentos (proteínas e dieta padrão). Entretanto, as proteínas provocaram uma discreta redução do Índice de Lee $[0.312(6,1)$ para $0.311(4,3)]$, e do peso [402 g (38.6) para 400 $\mathrm{g}$ (35.7)]. Quanto a dosagem de leptina, 60\% dos animais do grupo teste apresentaram redução de leptina $(<0,1 \mathrm{ng} / \mathrm{mL})$. Conclusão: As proteínas isoladas de tamarindo reduziram a leptina plasmática independente da perda de peso em ratos com obesidade, despontando como promissoras no estudo do tratamento da obesidade.

Palavras-chave: Obesidade; Índice de Lee; ratos Wistar. 


\section{Introdução}

A obesidade é considerada uma epidemia mundial, caracterizada pelo acúmulo excessivo de gordura corporal decorrente da hiperplasia e / ou hipertrofia das células adiposas (HAN; WU; LEAN, 2013; AFSHIN et al., 2017). Seu surgimento pode se dar por fatores genéticos, endócrinos e metabólicos ou então, influenciados por fatores externos - origem dietética, comportamental ou ambiental (ABESO, 2016).

Com essa complexa etiologia genética-ambiental, a obesidade apresenta uma gama de consequências para o organismo, podendo induzir o surgimento de desordens metabólicas, provocando distúrbios na homeostase hormonal, tendo como consequência, na maioria dos casos, o surgimento das mais variadas doenças, as quais se pode citar: hipertensão arterial, arteriosclerose, câncer, diabetes tipo 2, síndrome da insuficiência respiratória, embolia pulmonar, insuficiência cardíaca, infertilidade, entre muitas outras (SCHMIDT et al., 2011; JUNG; CHOI, 2014; WHO, 2016).

Sabe-se que existe uma relação estreita entre alimentação e o metabolismo hormonal envolvido no controle da fome e da saciedade (DOCKRAY, 2014). Um hormônio envolvido na modulação do apetite em longo prazo é a leptina. Secretada pelos adipócitos, responde pelo controle da ingestão alimentar, atuando em células neuronais do hipotálamo no sistema nervoso central. A leptina promove a redução da ingestão alimentar e o aumento do gasto energético, além de regular a função neuroendócrina e o metabolismo de carboidratos e de gorduras (TRIANTAFYLLOU; PASCHOU; MANTZOROS, 2016).

A ação de leptina tem sido estudada na saciedade, com pesquisas demonstrando que em animais e / ou indivíduos com obesidade, esses efeitos sacietogênicos não têm sido tão evidenciados quanto em eutróficos (MOOS; MCLAUGHLIN; BAILE, 1982; DUCA; ZHONG; COVASA, 2013; SÁINZ et al., 2015). Em relação à leptina, apesar dos indivíduos com obesidade apresentarem níveis elevados desse hormônio, uma vez que o mesmo é diretamente proporcional à quantidade de adipócitos, não é observado redução do apetite, o que sugere também uma condição de resistência (FREDERICH et al., 1995).

Sabe-se que o tecido adiposo subcutâneo ou branco é o principal local de produção da leptina, sendo produzida também, em pequenas quantidades, no epitélio gástrico fundo, intestino, placenta, músculo esquelético, epitélio mamário e cérebro (FLIER, 2004). Seu gene (gene ob) está localizado no cromossomo 7q31DNA e tem >15.000 pares de bases formado por três exons, tendo como produto final um hormônio composto por 167 aminoácidos, um polipeptídio não glicosilado com 16 kD, cuja função principal é regular o peso corporal, o metabolismo, a função reprodutiva e imunitária (ZHANG et al., 1994). No entanto, na obesidade, sua hipersecreção prejudica os efeitos já citados, uma vez que sua sensibilidade é reduzida (MARRADES; MARTÍNEZ; MORENO-ALIAGA, 2007), sendo de grande interesse tratamentos que promovam a sua redução para sua melhor atividade.

Observando a importância desses hormônios na gênese da obesidade, bem como a descoberta de sua atuação nessa condição fisiológica, é pertinente estudos com moléculas que atuem contribuindo para o melhor funcionamento dos mesmos. Dessa forma, pesquisas vêm sendo realizadas com proteínas bioativas, para as quais são observados efeitos benéficos à saúde, relacionados à modulação de respostas mediadas por proteínas, sejam enzimas ou hormônios, tais como propriedades: anti-coagulante, anti-inflamatório, anti-cancerígeno, anti-bacteriano, anti-fúngico e anti-obesidade (FANG et al., 2010; SCOOTT; TAGGART, 2010; MACHADO et al. 2013; RIBEIRO et al., 2015; SERQUIZ et al., 2016).

Na nutrição, estudar o potencial anti-obesidade e / ou sacietogênico de substâncias ou moléculas se reveste de grande importância, dado a relevância que a obesidade se apresenta para a saúde humana. Recentemente, estudos 
realizados pelo nosso grupo de pesquisa Reabilitação cardiovascular melhora capacidade funcional de pacientes cardiopatas após 3 anos de seguimento Nutrição e Substâncias Bioativas para Saúde (NutriSBioativoS), tem demonstrado que proteínas presentes nas sementes de tamarindo possuem atividades anti-obesidade e anti-inflamatória (RIBEIRO et al., 2015; CARVALHO et al., 2016).

Ribeiro et al. (2015) analisaram a influência dessas proteínas em modelo experimental de saciedade, e constataram que esse isolado proteico foi capaz de reduzir o consumo alimentar e o ganho de peso de animais eutróficos, ao elevarem a concentração plasmática da colecistoquinina (CCK). Já Carvalho et al. (2016) também testaram a atividade dessas mesmas proteínas em animais com obesidade e síndrome metabólica, condição típica de inflamação crônica, e verificaram que houve diminuição do consumo alimentar e do fator de necrose tumoral alfa (TNF-a), sem redução do ganho de peso.

Por essa razão, os modelos experimentais têm sido utilizados para estudar a obesidade, destacando-se dentre eles, os animais com obesidade induzida geneticamente (ZUCKER; ZUCKER, 1961; XU et al., 2016). Embora as mutações genéticas causem obesidade em animais, a ocorrência em humanos é rara (LUBRANO et al., 2003; CARROLL; VOISEY; DAAL, 2004). Assim, modelos que provocam obesidade com o uso de dietas (DIO, do inglês diet induced obesity) vêm sendo bastante valorizados, dados à etiologia da obesidade se apresentar de modo semelhante a do humano (MONCADA et al., 2016; KAMALAKKANNAN et al., 2016).

Desse modo, este estudo tem como finalidade avaliar o estado nutricional (medidas zoométricas), o peso e o efeito de proteínas isoladas das sementes de tamarindo sobre a concentração plasmática de leptina em ratos Wistar com obesidade, hormônio importante que está envolvido no controle da fome e do apetite.

\section{Metodologia}

Trata-se de um estudo experimental, o qual foi realizado com três grupos de ratos Wistar com obesidade para testar o efeito de proteínas isoladas de sementes de tamarindo.

O tamarindo foi adquirido comercialmente na cidade de Natal-RN, e suas sementes foram obtidas com a retirada dessas do interior do fruto e descascadas até obtenção apenas dos seus cotilédones.

Foram utilizados no experimento, ratos Wistar machos diagnosticados com obesidade de acordo com 0 Índice de Lee ( $\geq 300$ ) segundo Bernardis e Patterson (1968), mantidos no biotério da Universidade Potiguar (UNP) - Natal/ RN em condições padrão de luminosidade (12h/12h-claro/escuro) e temperatura $\left(23-25^{\circ} \mathrm{C}\right)$; água e alimento ad libitum. Todos os experimentos foram realizados em concordância com o Guia de Cuidado e Uso de Animais de Laboratório dos Institutos Nacionais de Saúde, e após aprovação do Comitê de Ética no Uso de Animais da UNP (CEUA - UNP) sob o número de protocolo: 012/2015.

As dietas utilizadas neste estudo foram a ração Labina ${ }^{\circledR}$ produzida pela Purina ${ }^{\circledR}$ do Brasil, sendo essa adquirida comercialmente, e a dieta experimental de alto índice e carga glicêmica (Dieta HGLI) produzida de acordo com Carvalho et al. (2017) no laboratório de Técnica em Dietética da Universidade Federal do Rio Grande do Norte (UFRN).

A metodologia utilizada para isolamento das proteínas das sementes foi estabelecida no Laboratório de Química e Função de Proteínas Bioativas (LQFPB) da UFRN e adaptada por Carvalho et al. (2016).

A partir do cotilédone das sementes obteve-se uma farinha de granulação fina, em seguida foi realizada a extração e obtenção das frações proteicas em diferentes faixas de saturação com sulfato de amônia. A fração proteica saturada na faixa de $30 \%-60 \%$ foi submetida a um processo de enriquecimento por cromatografia de afinidade em coluna 
de tripsina-sepharose (GE Health Care, Waukesha, Estados Unidos).

Para as avaliações do efeito das proteínas isoladas sobre o peso e índice de Lee, os ratos Wistar com obesidade foram distribuídos individualmente e casualmente em três grupos $(n=5)$, passando inicialmente por cinco dias de adaptação para se estabelecer as condições do experimento. Desse modo, após este período, os 15 animais foram submetidos por dez dias às dietas e aos tratamentos descritos abaixo:

Dieta HGLI mais água (Dieta HGI + $1 \mathrm{~mL}$ de água por gavagem), sendo denominado grupo sem tratamento (Dieta HGLI).

Dieta padrão mais água (Dieta padrão $+1 \mathrm{~mL}$ de água por gavagem), sendo denominado grupo tratamento padrão (Dieta Padrão).

Dieta HGLI mais proteínas (Dieta HGLI $+1 \mathrm{~mL}$ da proteína na concentração de $25 \mathrm{mg} / \mathrm{Kg}$ por gavagem), sendo denominado grupo tratamento teste (Dieta $\mathrm{HGI}+$ proteína isolada).

Todas as dietas (HGLI e padrão) foram oferecidas aos animais de todos os grupos por via oral, enquanto que a água e as proteínas foram administrados por gavagem oral.

No décimo primeiro dia de experimento os ratos foram, mais uma vez, avaliados quanto as medidas zoométricas e posteriormente sacrificados para a coleta do sangue que seguiram para análises plasmáticas de leptina.

Para a avaliação do estado nutricional (medidas zoométricas) dos animais foi realizado o cálculo do índice de Lee antes e após o tratamento.

No primeiro dia de experimento, antes da administração oral das dietas, foram realizadas as aferições e posterior cálculo do índice de Lee para confirmar a obesidade dos animais, e no último dia de tratamento, 1 hora após a administração dos tratamentos, também foram realizadas as mesmas aferições e cálculos.

$O$ índice de Lee se dá pela relação entre a raiz cúbica do peso corporal em gramas dividido pelo comprimento nasoanal em centímetros, tendo como ponto de corte valor $\geq 300$ como diagnóstico de obesidade de acordo com Bernardis e Patterson (1968). Todas as medidas foram conduzidas por pesquisadores treinados, visando atender aos critérios de autenticidade científica, como recomendado por Novelli et al. (2007).

Índice de Lee $=[3 \sqrt{ }$ Peso $(\mathrm{g}) / \mathrm{CNA}(\mathrm{cm})]$

Ao término dos tratamentos, os 15 animais foram colocados em jejum por 8 horas, anestesiados com Tiletamina (cloridrato) $250 \mathrm{mg}$ e Zolazepam (cloridrato) $250 \mathrm{mg}$ e em seguida eutanasiados. 0 sangue foi coletado pela veia hepática. $O$ soro foi separado por centrifugação a 3000 rpm por 10 minutos e utilizado para a determinação da concentração da leptina no plasma usando o Kit Rat Leptin ELISA (Millipore® - EZRL-83K).

Os dados foram analisados para a normalidade usando o teste de Shapiro Wilk. A variável do índice Lee não foi paramétrica e o teste Two-way Anova com o teste post hoc de Dunn foi utilizado para verificar as diferenças entre os grupos estudados. As diferenças nas concentrações de leptina foram realizadas pelo teste Pearson Chi-Square. Os dados foram analisados usando Graph Pad Prism, versão 5.0 (Graph Pad Software, San Diego, CA). Para todos os testes, p valores inferiores a 0,05 foram considerados significativos.

\section{Resultados}

De acordo com o índice de Lee todos os animais estudados estavam com obesidade no início do experimento, e ao serem tratados com as proteínas e a dieta padrão, esse índice não se alterou em ambos os tratamentos, mantendo-os no mesmo estado de obesidade. Entretanto, os animais que receberam as proteínas, mesmo consumindo a dieta HGLI, apresentaram uma discreta redução do Índice de Lee $[0.312(6,1)$ para $0.311(4,3)]$, enquanto que os grupos de animais 
que consumiram apenas a dieta HGLI $(0.306 \pm 5,3$ para $0.309 \pm 4,8)$ e a dieta nutricionalmente adequada $[0.310(5,6)$ para $0.311(11,7)$ ] apresentaram a tendência inversa no mesmo índice, sendo esse aumento mais expressivo no grupo que se alimentou durante os 10 dias de experimentos apenas com a dieta HGLI, considerado o grupo controle, sem tratamento.

Os dados relacionados ao ganho de peso apresentaram-se de modo semelhante ao índice de Lee. Os animais que receberam as proteínas (402(38.6) para 400(35.7)] e a dieta nutricionalmente adequada [(399(29.4) para 39815,7)] apresentaram uma discreta redução do ganho do peso, já os animais que consumiram apenas a dieta HGLI [(400(35.7) para 406(27.7)] constatou-se moderado aumento, sem diferenças significativas, como demostrado também pelo índice de Lee, durante os 10 dias de experimentos.

A tabela 1 apresenta o percentual de animais com concentrações detectáveis da leptina plasmática referente aos três grupos analisados no estudo. Sendo assim, pode-se verificar que $60 \%$ dos animais do grupo que receberam como tratamento as proteínas apresentaram concentrações plasmáticas de leptina menor que $(<) 0,1 \mathrm{ng} / \mathrm{mL}$, sendo esse o valor mínimo detectável na metodologia utilizada. Enquanto que o grupo que foi tratado, de modo convencional, com uma dieta nutricionalmente adequada para ratos Wistar, apresentou apenas $20 \%$ dos animais, pertencentes a esse grupo, com concentrações de leptina $<0,1 \mathrm{ng} / \mathrm{mL}$. Já o grupo que não recebeu tratamento e se manteve consumindo a mesma dieta que foi usada para induzir a obesidade, na intenção de mantê-la, apresentou em $100 \%$ dos seus animais as concentrações plasmáticas acima de $(\geq) 0,1 \mathrm{ng} / \mathrm{mL}$.

Tabela 1. Valores plasmáticos de leptina dos ratos Wistar com obesidade pertencentes aos três grupos submetidos aos diferentes tratamentos durante 10 dias de experimento.

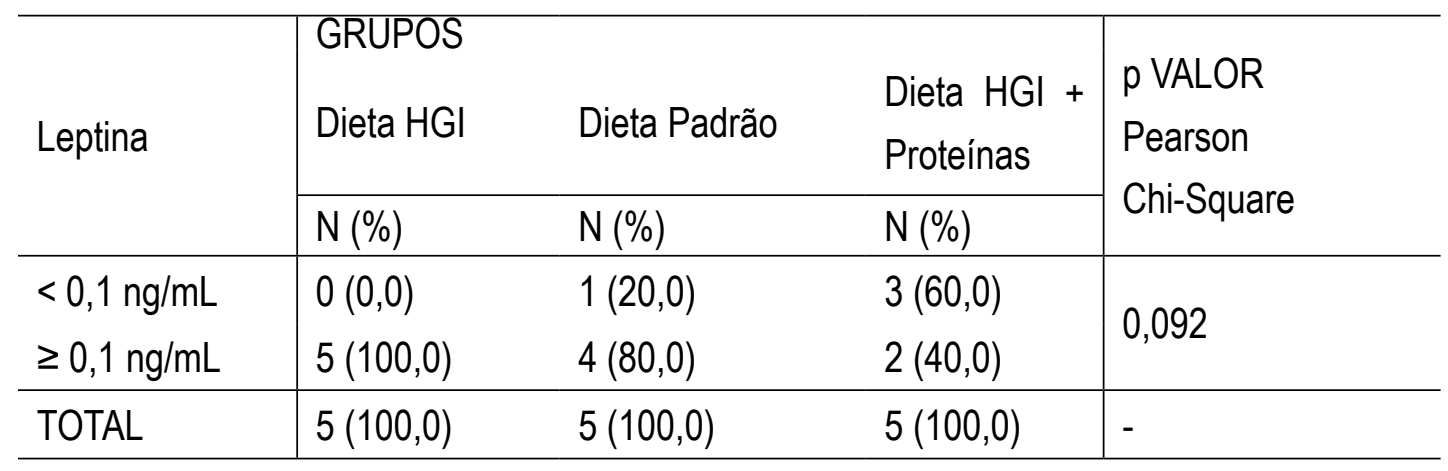

Grupos: Dieta HGLI mais água (Dieta HGI $+1 \mathrm{~mL}$ de água por gavage); Dieta padrão mais água (Dieta padrão $+1 \mathrm{~mL}$ de água por gavage); Dieta HGLI mais Proteínas (Dieta HGI $+1 \mathrm{~mL}$ de proteínas na concentração de $25 \mathrm{mg} / \mathrm{Kg}$ por gavage). Dieta HGLI: Dieta de alto índice e carga glicêmica - Mistura composta pela ração Labina ${ }^{\circledR}$, leite condensado e açúcar (1:1:0.21); Dieta padrão: Ração Labina ${ }^{\circledR}$.

\section{Discussão}

Estudos prévios do nosso grupo de pesquisa, NutriSBioativoS, têm demonstrado que proteínas isolada das sementes de tamarindo apresentam atividade sacietogênica e anti-inflamatória (RIBEIRO et al., 2015, CARVALHO et al., 2016). Desse modo, essas proteínas foram novamente obtidas das sementes de tamarindo e neste estudo apresentou as mesmas características referidas por Ribeiro et al. (2015) e Carvalho et al. (2016). Assim, elas foram oferecidas por gavagem para um dos três grupos de animais com obesidade, sendo avaliadas quanto a sua influência no estado nutricional de obesidade, utilizando como ferramenta avaliativa o Índice de Lee (BERNARDIS; PATTERSON, 1968), critério que se assemelha ao IMC utilizado como padrão de diagnóstico do estado nutricional em humanos (ABESO 2016). 
Neste experimento foi observado que todos os animais encontravam-se com obesidade de acordo com 0 índice de Lee, no início do tratamento. Após o período de tratamento, ainda foi observado obesidade com base no mesmo índice, em todos os animais participantes do experimento, porém, para os animais que fora administrado as proteínas, foi possível constatar uma discreta redução no ganho de peso quando comparado aos animais sem tratamento ou submetidos ao tratamento convencional.

Os dados mostram que mesmo os animais consumindo uma dieta altamente desequilibrada, capaz de provocar obesidade e suas comorbidades associadas (CARVALHO et al., 2016), quando recebem as proteínas reduz moderadamente, mas sem diferença significativa entre os grupos, como o índice de Lee, no entanto ressalta-se que 0 estudo ocorreu em apenas dez dias. Considerando que essas mesmas proteínas não influenciaram a perda de peso de ratos Wistar com obesidade, mas no entanto levou a redução do consumo alimentar (CARVALHO et al., 2016) hipotetizase a necessidade de um maior período de tempo para se observar as mudanças significativas nesse índice zoométrico.

Também foi analisado e avaliado a influência dessas proteínas nas concentrações plasmáticas de Leptina. Já é consenso pela literatura científica que grande parte dos indivíduos que se encontram no estado de obesidade apresentam níveis sérios de leptina elevados, sendo estes proporcionais a sua massa de tecido adiposo (CONSIDINE et al., 1996; MAFFEl et al., 1995).

Era esperado neste estudo, que os animais com obesidade, apresentassem em sua totalidade, concentrações plasmáticas de leptina alta, já que, como visto na literatura, essa condição leva a hiperleptinemia (FREDERICH et al., 1995; SÁINZ et al., 2015). Entretanto, foi observado que o tratamento com as proteínas de sementes de tamarindo provocaram redução dessas concentrações em $60 \%$ dos animais, ainda com obesidade e consumindo a dieta HGLI, a mesma usada na indução da obesidade pelos animais utilizados nesta pesquisa e consumida pelo grupo para manter a obesidade, sem tratamento.

Ainda, foi demostrado que os animais submetidos ao tratamento convencional com dieta padrão, nutricionalmente adequada, reduziram apenas em $20 \%$ dos animais as concentrações de leptina. Ademais, no estudo de Carvalho et al. (2016), essas proteínas já haviam apresentando efeito na redução do TNF-a, independente da redução do peso, uma molécula também produzida pelo tecido adiposo. Esses achados com as proteínas obtidas das sementes de tamarindo são inéditos e se revestem de grande importância científica dado ao efeito inesperado sobre a redução de leptina plasmática independente da perda de peso, ou melhor, de tecido adiposo.

\section{Conclusão}

As proteínas isoladas das sementes de tamarindo não diminuíram de modo significativo o ganho de peso e o estado de obesidade dos animais analisados neste estudo. No entanto, foi capaz de reduzir discretamente esses parâmetros zoométricos e surpreendentemente atuaram reduzindo a concentração plasmática de leptina independente dos efeitos sobre o peso e consequente estado de obesidade.

\section{Referências bibliográficas}

AFSHIN A.; FOROUZANFAR M. H., REITSMA M. B., SUR P., ESTEP K., LEE A., et al. Health effects of overweight and obesity in 195 countries over 25 years. The New England Journal of Medicine, v. 377, n. 1, p. 13-27. 2017. Associação Brasileira para o Estudo da Obesidade e da Síndrome Metabólica. Diretrizes brasileiras de obesidade 2016/ ABESO - Associação Brasileira para o Estudo da Obesidade e da Síndrome Metabólica. 4. ed. São Paulo: ABESO; 2016. 
p.1-186.

BERNARDIS, L. L.; PATTERSON, B. D. Correlation between 'Lee index' and carcass fat content in weanling and adult female rats with hypothalamic lesions. Journal of Endocrinology, v. 40, n. 4, p. 527-528, 1968.

CARROLL, J.; VOISEY, J.; DAAL, A. V. Mouse models of obesity. Clinics in Dermatology, v. 22, n. 4, p. 345-349, 2004. CARVALHO, F. M. C.; COSTA, I. S.; SANTOS, J. P. S. O.; OLIVEIRA G. S.; SANTOS, J.; SILVA, F. K. B.; BORTOLIN, R. H.; SILBIGER, V. N.; NEVES, R. A. M.; MACIEL, B. L. L.; SANTOS, E. A.; MORAIS, A. H. A. A high glycemic and load index pellet-diet induces obesity, hyperglycemia, dyslipidemia and increased visceral and subcutaneous adipose tissue PPARy expression in Wistar rats. Food Chemistry, submitted, 2017.

CARVALHO, F. M. C.; LIMA, V. C. O.; COSTA, I. S.; MEDEIROS, A. F.; SERQUIZ, A. C.; LIMA, M. C. J. S.; UCHÔA, A. F.; SANTOS, E. A.; MORAIS, A. H. A Trypsin Inhibitor from Tamarind Reduces Food Intake and Improves Inflammatory Status in Rats with Metabolic Syndrome Regardless of Weight Loss. Nutrients, v. 8, n. 544, p. 1-14, 2016.

CONSIDINE, R. V.; SINHA, M. K.; HEIMAN, M. L.; KRIAUCIUNAS, A.; STEPHENS, T. W.; NYCE, M. R.; OHANNESIAN. J. P.: MARCO, C. C.: MCKEE, L. J.; BAUER, T. L. Serum immunoreactive leptin concentrations in normal-weight and obese humans. New England Journal of Medicine, v. 334, n. 5, p. 292-295, 1996.

DOCKRAY, G. J. Gastrointestinal hormones and the dialogue between gut and brain. Journal of Physiology, v. 14, n. 592, p. 2927-2941, 2014.

DUCA, F. A.; ZHONG, L.; COVASA, M. Reduced CCK signaling in obese-prone rats fed a high fat diet. Hormones and Behavior, v. 64, n. 5, p. 812-817, 2013.

FANG, E. F.; WONG, J. H.; BAH, C. S. F.; LIN, P.; TSAO, S. W.; NG, T. B. Bauhinia variegate var. variegate trypsin inhibitor: From isolation to potencial medicinal applications. Biochemical and Biophysical Research Communications, v. 396, n. 4, p. 806-811, 2010.

FLIER, J. S. Obesity Wars: Molecular Progress Confronts an Expanding Epidemic. Cell, v. 116, n. 23, p. 337-350, 2004. FREDERICH, R. C.; HAMANN, A.; ANDERSON, S.; L"OLLMANN, B.; LOWELL, B. B.; FLIER, J. S. Leptin levels reflect body lipid content in mice: evidence for diet-induced resistance to leptin action. Nature Medicine, v. 1, n. 12, p. 13111314, 1995.

HAN, T. S.; WU, F. C. W.; LEAN, M. E. J. Obesity and Weight management in the elderly: a focus on men. Best Practice \& Research Clinical Endocrinology \& Metabolism, v. 27, n. 4, p. 509-525, 2013.

JUNG, U. J.; CHOI, M. S. Obesity and its metabolic complications: the role of adipokines and the relationship between obesity, inflammation, insulin resistance, dyslipidemia and nonalcoholic fatty liver disease. International Journal of Molecular Sciences, v. 15, n. 4, p. 6184-6223, 2014.

KAMALAKKANNAN, S.; TIRUPATHI, P. B. P.; KALAISELVI, S.; ARUNACHALAM, S.; ACHIRAMAN, S. Emu oil decreases atherogenic plaque formation in cafeteria diet-induced obese rats. Journal of the Science of Food and Agriculture, $v$. 96, n. 9, p. 3063-3068, 2016.

LUBRANO, C. B.; CAVAZOS, M.; DUBERN, B.; SHAPIRO, A.; STUNFF, C. L.; ZHANG, S.; PICART, F.; GOVAERTS, C.; FROGUEL, P.; BOUGNERES, P.; CLEMENT, K.; VAISSE, C. Molecular genetics of human obesity-associated MC4R mutations. Annals of the New York Academy of Sciences, v. 994, p. 49-57, 2003.

MACHADO, R. J. A.; MONTEIRO, N. K. V.; MIGLIOLO, L.; SILVA, O. N.; PINTO, M. F. S.; OLIVEIRA, A. S.; FRANCO, O. L.; KIYOTA, S.; BEMQUERER, M. P.; UCHOA, A. F.; MORAIS, A. H.; SANTOS, E. A. Characterization and pharmacological properties of a novel multifunctional kunitz inhibitor from Erythrina velutina seeds. PloS One, v. 8, n. 5, p. 1-14, 2013. 
MAFFEI, M.; HALAAS, J.; RAVUSSIN, E.; PRATLEY, R. E.; LEE, G. H.; ZHANG, Y.; FEI, H.; KIM, S.; LALLONE, R.; RANGANATHAN, S.; KERN P. A.; FRIEDMAN, J. M. Leptin levels in human and rodent: measurement of plasma leptin and ob RNA in obese and weightreduced subjects. Nature Medicine, v. 1, p. 1155-61, 1995.

MARRADES, M. P.; MARTíNEZ, J. A.; MORENO-ALIAGA, M. J. Differences in short-term metabolic responses to a lipid load in lean (resistant) vs obese (susceptible) young male subjects with habitual high-fat consumption. European Journal of Clinical Nutrition, v. 61, p. 166-174, 2007.

MONCADA, R.; RODRÍGUEZ, A.; BECERRIL, S.; MÉNDEZ, L. G.; VALENTÍ, V.; RAMÍREZ, B.; CIENFUEGOS, J. A.; FERNÁNDEZ, S.; CATALÁN, V.; GÓMEZ-AMBROSI, J.; FRÜHBECK, G. Sleeve Gastrectomy Decreases Body Weight, Whole-Body Adiposity, and Blood Pressure Even in Aged Diet-Induced Obese Rats. Obesity Surgery, v. 26, n. 7, p. 1549-1558, 2016.

MOOS, A. B.; MCLAUGHLIN, C. L.; BAILE, C. A. Effects of CCK on Gastrointestinal Function in Lean and Obese Zucker Rats. Peptides, v. 3, n. 4, p. 619-622, 1982.

NOVELLI, E. L. B.; DINIZ, Y. S.; GALHARDI, C. M.; EBAID, G. M. X.; RODRIGUES, H. G.; MANI, F.; FERNANDES, A. A.; CICOGNA, A. C.; NOVELLI FILHO, J. L. Anthropometrical parameters and markers of obesity in rats. Journal of laboratory animal, v. 41, n. 1, p. 111-119, 2007.

RIBEIRO, J. A.; SERQUIZ, A. C.; SILVA, P. F.; BARBOSA, P. B.; SAMPAIO, T. B.; ARAÚJO, R. F. J.; OLIVEIRA, A. S.; MACHADO, R. J.; MACIEL, B. L.; UCHÔA, A. F.; SANTOS, E. A. Tamarindus indica L. seed trypsin inhibitor reduce weight gain, food consumption and increases plasmatic cholecystokinin levels. Clinics, v. 70, n. 2, p. 136-143, 2015.

SÁINZ, N.; BARRENETXE, J.; MORENO-ALIAGA, M. J.; MARTÍNEZ, J. A. Leptin resistance and diet-induced obesity: central and peripheral actions of leptin. Metabolism Clinical and Experimental, v. 64, n. 1, p. 35-46, 2015.

SCHMIDT, M. I.; DUNCAN, B. B.; AZEVEDO E SILVA, G.; MENEZES, A. M.; MONTEIRO, C. A.; BARRETO, S. M.; CHOR, D.; MENEZES P. R. Chronic non-communicable diseases in Brazil: burden and current challenges. Lancet, v. 337, n. 9781, p. 1949-1961, 2011.

SCOOTT, C. J.; TAGGART, C. C. Biologic protease inhibitors as novel therapeutic agents. Biochimie, v. 92, n. 11, p. 1681-1688, 2010.

SERQUIZ, A. C.; MACHADO, R. J. A.; SERQUIZ, R. P.; LIMA, V. C. O.; CARNEIRO, M. A. A.; MACIEL, B. L. L.; UCHÔA, A. F.; SANTOS, E. A.; MORAIS, A. H. Supplementation with a new trypsin inhibitor from peanut is associated with reduced fasting glucose, weight control, and increased plasma CCK secretion in an animal model. Journal of Enzyme Inhibition and Medicinal Chemistry, v. 31, n. 6, p. 1-9, 2016.

TRIANTAFYLLOU, G. A.; PASCHOU, S. A.; MANTZOROS, C. S. Leptin and Hormones: Energy Homeostasis. Endocrinology Metabolism Clinics of North America, v. 45, n. 3, p. 633-645, 2016.

World Health Organization. Obesity and overweight: fact sheet. Disponivel em: <http://www.who.int/mediacentre 1 factsheets/fs311/en/>. Acesso: 18 de dezembro de 2016.

XU, X.; WANG, J.; LI, L.; WANG, C.; LI, W.; ZHANG, Q.; YANG, L. The role of obestatin in Roux-en-Y gastric bypass surgery in the obese, type 2 diabetes Zucker rat. Diabetes Research and Clinical Practice, v. 119, p. 57-64, 2016.

ZHANG, Y.; PROENCA, R.; MAFFEI, M.; BARONE, M.; LEOPOLD, L.; FRIEDMAN, J M. Positional cloning of the mouse obese gene and its human homologue. Nature, v. 372, n. 6505, p. 425-432, 1994.

ZUCKER, L. M.; ZUCKER, T. F. Fatty, a new mutation in the rat. Journal of Heredity, v. 52, n. 6, p. 275-278, 1961. 